\title{
Modelamiento de las elasticidades del índice de tasa de cambio real de bienes transables-deuda total e índice de términos de intercambio en Colombia a través de modelos vec
}

\author{
Modeling of the elasticities of the real exchange rate index of \\ transable property-total debt and index of exchance terms in \\ Colombia through vec models \\ Natalia Galvis ${ }^{\mathrm{a}}$
natilla12@gmail.com \\ Juliana Ahumada ${ }^{b}$ \\ julianaahumadah@usantotomas.edu.co \\ Heivar Rodríguez ${ }^{\mathrm{C}}$ \\ heivarrodriguez@usantotomas.edu.co
}

\begin{abstract}
Resumen
El objetivo de este trabajo es analizar los efectos y relaciones de tres variables de la economía internacional expresadas en elasticidades como el Índice de Términos de Intercambio (TDI), el Índice de Tipo de Cambio Real como estimador el Índice de Precios del Productor (ITCR_IPP) y el Índice de la Deuda Total Colombiana en dólares (TDPYP), las cuales son susceptibles a los choques externos e internos de la política monetaria, cambiaria y fiscal, evaluadas a través de un modelo de series de tiempo multivariada conocido como VEC para los periodos comprendidos entre enero del 2009 a diciembre del 2018, donde se encontró una relación directa entre las variables TDI y TDPYP y una relación inversa entre TDI y ITCR_IPP.
\end{abstract}

Palabras clave: Modelos macroeconómicos, Dinámica económica, Análisis económico, Modelo VEC, Cointegración. (Clasificación JEL: B22, C32, C58).

\begin{abstract}
\footnotetext{
${ }^{a}$ Economísta. Especialista en Estadistica y en Ciencias Tributarias.

${ }^{\mathrm{b}}$ Estudiante. Facultad de Estadística. Universidad Santo Tomas.

${ }^{\mathrm{c}}$ Docente. Facultad de Estadística. Universidad Santo Tomas.
}

The objective of this article is analyze the effects and relationships of three variables of the international economy expressed in elasticities such as the Exchange Terms Index (TDI), the Real Exchange Rate Index as an estimator of the Producer Price Index (ITCR_IPP) and the Total Colombian Debt Index in dollars 
(TDPYP), which are susceptible to external and internal monetary, exchange and fiscal policy shocks, evaluated through a multivariate time series model known as VEC for periods from January 2009 to December 2018, where a direct relationship between the TDI and TDPYP variables and an inverse relationship between TDI and ITCR_IPP was found.

Keywords: Macroeconomic models, Economic dynamics, Economic analysis, VEC model, Cointegration.(JEL Classification: B22, C32, C58.).

\section{Introducción}

En Colombia es importante el análisis de relaciones entre variables, especialmente variables de tipo macroeconómico, como por ejemplo tres de las principales variables como lo son: 1) Elasticidad del índice de términos de intercambio, 2) Elasticidad del índice de tipo de cambio real como estimador el índice de precios del productor (bienes transables) y 3) Elasticidad del índice de la deuda total colombiana en USD, como variables susceptibles a choques externos e internos de política monetaria, cambiaria y fiscal; valoradas en un contexto de modelos robustos de estadística de series de tiempo multivariadas, las pruebas serán evaluadas al $5 \%$ para los periodos mensuales comprendidos entre Enero del 2009 a Diciembre del 2018, en los cuales serán estimadas como elasticidades para analizar la variación que tienen una respecto a la otra, de esta forma encontrar la sensibilidad entre las variables y una posible dependencia, por lo tanto Existe una relación más allá de la teoría económica que sea posible evaluar las elasticidades del índice de términos de intercambio, el índice de tipo de cambio real como estimador el índice de precios del productor (bienes transables) y el índice de la deuda total colombiana en USD a través de sus datos en las series macroeconómicas?

Así mismo Misas (2000) para analizar los términos de intercambio, el producto real, el gasto publico real, y la base monetaria nominal para el caso colombiano, integraron el análisis de los ciclos económicos con el comportamiento del crecimiento económico para Colombia de los años 20 al 1997, y el análisis de los choques sean positivos o negativos a partir de cambios en la oferta con el inicio y difusión en el tiempo de las fluctuaciones causadas por estos. En este trabajo los autores lograron una extensión de la serie del producto indicada como la principal fuente de información respecto al crecimiento de la economía, concluyendo consigo que las principales coyunturas fuesen explicadas y soportadas por el conocimiento previo al análisis siendo la magnitud de las brechas sensibles al tamaño de la muestra, lo que claramente indica que los problemas de medición que se han presentado a través del tiempo de las variables económicas del país como principal limitante en análisis.

Igualmente Echavarría et al. (2009) a través de un modelo VAR estructural (SVAR) en el corto plazo evalúan el impacto de las intervenciones cambiarias y de la política monetaria sobre la tasa de cambio, al estimar dicho modelo encuentran que la metodología empleada no cumple con lo propuesto inicialmente que es analizar 
en su conjunto los dos tipos de política, para ello indican que no tuvieron en cuenta una variable que recogiera las expectativas de inflación o la expectativa de una tasa de cambio futura, y concluyen que es necesario nuevas investigaciones en el campo. A su vez analizan lo encontrado con las variables estudiadas para la tasa de cambio nominal encuentran que se devalúa cuando se interviene el mercado cambiario, cuando se presenta aumentos en la inflación y se reducen los términos de intercambio, pero el impacto suele variar, adicional a esto se perciben incrementos en la tasa de cambio los primeros meses dada la inflación y compras de divisas, en cambio la tasa de cambio se ve afectada por los términos de intercambio para un mes 11, el impacto sobre la tasa de cambio nominal y de los choques nominales en políticas de intervención, política monetaria y la demanda de dinero de acuerdo a lo indicado por la descomposición de la varianza se comporta de manera similar a los choques reales que se ven en cambios de nivel de producto y términos de intercambio estos últimos siendo más rápidos que los anteriormente descritos. A pesar que el modelo no funciona para pronosticar o realizar análisis más profundos ayuda al análisis del comportamiento de variables importantes en el sentido macroeconómico.

Con respecto a los términos de intercambio Clavijo et al. (n.d.) generan dos modelos teóricos en el contexto uruguayo que demuestran que el salario real depende positivamente de la evolución de los términos de intercambio teniendo una relación positiva en el largo plazo, esta relación es sustentada por los modelos tradicionales de comercio a través de la intensidad relativa de factores, en especial intensivos en mano de obra lo que se asegura que es una economía en vías de desarrollo ya que se ajusta a los choques exógenos de los precios relativos de las commodities; el modelo de corte microeconómico no hace supuestos sobre la especialización de los factores de producción, ni sobre la dotación inicial de factores, los principales resultados indican que la evolución del salario real depende de los términos de intercambio de los productos que se comercializan con otros países, esto conlleva a que las políticas que están dirigidas a los consumidores (asalariados) para aumentar el poder adquisitivo (compra) son restringidas dado que depende de los factores externos.

Por otra parte Bigio \& Salas (2006) en la política monetaria y el tipo de cambio real para la economía peruana, enfocaron su trabajo en explorar las variaciones que tienen las posiciones de la política monetaria y el tipo de cambio real para una economía parcialmente dolarizada en temas como la inflación, y el producto teniendo como resultado que la política monetaria tiene efectos fuertes en el producto en épocas de recesión o crecimiento lento en las economías, y en épocas de prosperidad o auge la relación es positiva para la inflación, en el caso de la relación que tiene la tasa de cambio real sugiere depreciaciones que contraen la economía en el corto plazo lo que equivaldría a lo conocido como efecto hoja de balance es decir que existe efecto de tipo patrimonio (negativo) y/o de competitividad (positivo) que llegan a ser nocivos para épocas de recesión, mencionan parcialmente las implicaciones de un posible análisis de la curva de Philips pero no se realiza lo cual hubiese sido de gran ayuda para el análisis de forma más concreta los efectos de la política monetaria e incluso un análisis más profundo del efecto hoja de balance.

Comunicaciones en Estaística, diciembre 2019, Vol. 12, No. 2 
Por su parte Calderón (2006) quien analiza el tipo de cambio real (TCR) para la economía Chilena, utiliza la cointegración de Johansen y un análisis de robustez para la TCR con una canasta ampliada, entre los principales hallazgos de este estudio se encuentra a largo plazo una relación entre la tasa de cambio real y los términos de intercambio y el gasto del gobierno, así mismo los términos de intercambio tiene una relación significativa sobre la tasa de cambio real, también encuentran que la depreciación del peso chileno se ve reflejada en un mayor endeudamiento externo y una caída en los términos de intercambio, así mismo busca un modelo para evaluar el tipo de cambio de tendencia de la TCR y el modelo cambia de acuerdo a la muestra empleada, el análisis es muy enriquecedor en cuanto al análisis histórico de la tendencia de la variable evaluada, pero el estudio no va más allá de ser un análisis descriptivo.

Las ideas expuestas por Rodríguez (2011) analiza para el caso colombiano el precio promedio mensual del petróleo WTI de referencia e IPC de Colombia, variables a las que se les debió transformar para cumplir con las especificaciones del modelo VAR, se llega a la conclusión que en Colombia el precio del petróleo (WTI) ha sido la principal causa de la importación de la inflación, adicional a esto al ser un país exportador de petróleo el precio del mismo afecta gravemente a la economía, que se ve reflejado a través del costo de vida evaluado como IPC o "inflación" , este fenómeno va ligado a países exportadores ya que incita al alza, en cambio para los países importadores este se puede controlar a través del control de precios, el análisis se puede complementar con la inclusión de otras variables económicas que pueda influenciar en el comportamiento del precio del petróleo.

De acuerdo con (Arteaga et al. 2012) los cuales analizan la tasa de cambio real (TCR) a través del análisis Bassala - Samuelson la cual permite determinar la temporalidad donde la serie se encuentre alejada de la relación de cointegración, este análisis se centra en los movimientos de los activos a corto plazo y el consumo a mediano plazo analizado por los fundamentales, y se complementa el análisis de largo plazo con la metodología VEC para evaluar la TCR respeto a las variables denominadas como fundamentales en la literatura económica como lo son TRM, gasto, productividad, términos de intercambio, consumo público, tasa de interés de los bonos del tesoro, entre otras, dando consigo apreciaciones y depreciaciones entre las interacciones de las variables, una de las más importantes es la tendencia entre las variables de TCR y los términos de intercambio que desde 2003 siguen la misma tendencia hasta la fecha y que las variaciones del consumo del gobierno influye en la dinámica de dicha variable, los autores introducen la posibilidad de ampliar el análisis a un modelo panel de contraste entre países lo cual sería muy interesante de evaluar.

Al comparar estas evidencias con Otero (1997), se encuentra que él analiza la tasa de cambio real (TCR) junto con el precio externo del café, el saldo de la deuda externa (privada), el gasto corriente del gobierno central para lo cual incrementos de estas variables se ve reflejado en una apreciación de la variable principal analizada

y se deprecia ante respuestas e intervenciones de política monetaria como lo es las medidas proteccionistas, adicional a esto encontró que no depende de desequili- 
brios del mercado monetario, el periodo analizado por el autor es muy corto pero hace un importante análisis de las relaciones de algunas variables macroeconómicas importantes de análisis.

A estos elementos se le suman el análisis realizado por Licandro \& Masoller (2000) donde se evalúa la composición optima de las divisas que conforman la deuda pública uruguaya, a través del análisis entre el sesgo de la deuda en moneda extranjera y el riesgo presupuestal que está implícito en los instrumentos en dólares. La deuda no está compuesta solo de un valor que cuantifica la responsabilidad de pago también implícitamente esta evaluada en términos de la tasa de inflación, la tasa de depreciación real, la tasa de interés internacional, la tasa de variación del salario real y la tasa de crecimiento del producto para lo cual utilizan los modelos VAR para el análisis correspondiente; uno de los principales hallazgos es el que involucra a la TCR con una alta variabilidad causada por el abandono de la "Tablita" un sistema de paridad cambiaria lo que produjo una variabilidad excesiva en el presupuesto público, adicional a esto encontraron que el portafolio de la deuda provoca que el costo de la misma sea pro-cíclico y es catalogado como perverso porque cuando a la economía nacional está en un mal momento, este se comporta peor.

Para Colombia Vásquez \& Basto, (2004) analizaron la deuda a través de un VAR estructural (SVAR) encontrando consigo una desviación del nivel de deuda de su nivel de equilibrio, esto puede verse justificado en gran parte por la relación que existe con las variaciones en la inflación, tasa de cambio, tasa de interés interna, y la tasa de interés externa (algo anteriormente justificado por Licandro \& Masoller (2000)) y congruente con un análisis estructural, por ende estas variables y su volatilidad corresponden a variables de "riesgo" como lo indican los autores para la deuda, ante esto el análisis de dichas variables se convirtió en algo imperante encontrando que las volatilidades de la inflación y tasa de cambio son las que más afectan la deuda total representado con una mayor volatilidad. En cuanto a la composición de la deuda total se evidencio que el desequilibrio de la deuda externa $(59 \%)$ fue la que más aporto en la distancia entre el equilibrio y los datos reales, en comparación con la deuda interna (41\%), a lo que los autores hacen un llamado a un ajuste para revertir la tendencia imperante, adicional a esto se hace necesario ampliar el análisis a el costo por el servicio de la deuda.

Las variables como la elasticidad del índice de la tasa de cambio real, estimada a través de los datos calculados por el Banco de la republica a través del ITCR_IPP (Índice de tasa de cambio real para el índice de precios del productor) como lo indica Fernández (2013) tiene la posibilidad de ayudar a las economías en épocas de crisis no directamente sino a través de los efectos que tiene en la cuenta corriente en donde se encuentra la balanza comercial, la balanza de pagos y en las reservas internacionales, es decir que esta variable ayuda a mitigar los efectos de los choques externos negativos tanto en el sector real como en el financiero disminuyendo la fluctuación e incentivando la producción e incluso la inversión.

El presente artículo busca encontrar la relación existente entre las elasticidades del índice de tasa de cambio real de bienes transables, deuda total, e índice de 
términos de intercambio en Colombia. A lo largo del documento se presentan las variables y la metodología para determinar el tipo de relación existente, la cual se estima con la Función Impulso Respuesta del modelo VEC (usando el software estadístico EVIEWS 9.0). El periodo de estudio comprende desde enero del 2009 hasta diciembre del 2018.

\section{Desarrollo}

La metodología de los modelos de vector auto regresivos (VAR) por Sims (1980); son utilizados cuando se requiere caracterizar las relaciones simultaneas de una serie de variables, adicional a esto tienen la ventaja que no incurren en problemas de especificación (Lutkepohl (2005)).

Dentro de este marco Pindyck \& Rubinfeld (2001) afirman que las auto regresiones vectoriales (VAR) proporcionan el medio para especificar una estructura más dinámica en un modelo económico.

En efecto Andrade et al. (2007) indican que podrán ser utilizados este tipo de modelos en la estimación de parámetros de interés útiles a la formulación de políticas, pues permiten obtener la descomposición de la varianza y las funciones impulso respuesta, con estos modelos se pueden explicar: El tiempo de reacción de las respuestas a los choques, la dirección, el patrón y la duración de estas respuestas, y la intensidad de interacción entre las diversas variables contenidas en el VAR.

Los modelos VEC, están dentro de los modelos VAR anteriormente descritos, incluyen la corrección de errores (MCE), el término de corrección de errores corresponde a combinaciones lineales, que podrían emplearse para predecir como un VAR pero a través de la función impulso y respuesta para conocer el comportamiento de las series, este modelo tiene otras implicaciones como es el contraste de cointegración de Johansen que puede hacer este cálculo sin importar el número de series que se tengan en el modelo, puede analizar la exogeneidad de las variables en el VAR (Medina (2015)), ya que tienen en cuenta los vectores de cointegración para dicho modelo.

\section{Elasticidad del índice de tipo de cambio real evaluado a través del Índice de Precios del Productor bienes transables}

El índice del tipo de cambio real evaluado a través del índice de precios del productor equivalente a los bienes transables de la economía (ITCR_IPP), hace parte de las series disponibles del Banco de la Republica de Colombia en la Gerencia técnica, la cual corresponde a la relación del tipo de cambio nominal del peso colombiano con respecto al conjunto de monedas externas, utiliza las ponderacio- 
nes totales ${ }^{1}$ considerando el IPP ${ }^{2}$ Índice de precios del productor como deflactor, lo que indica que su canasta la conforman bienes transables y refleja mejor los cambios en los precios relativos de los bienes, dicha variable fue transformada por logaritmo dejándola expresada como elasticidad ${ }^{3}$.

\section{Elasticidad del índice de términos de intercambio}

El índice de términos de intercambio (es la razón entre el precio de las exportaciones y el precio de sus importaciones) es el total de los términos de intercambio proporcionado por las estadísticas aduaneras DIAN- DANE, a través de la metodología de ((Garavito et al. 2011)), la cual ha sido transformada a logaritmo dejándola expresada como elasticidad ${ }^{4}$.

\section{Elasticidad del índice de la deuda total colombiana en dólares}

El índice de la deuda total colombiana en dólares de acuerdo a la descripción del Banco de la república está compuesto por el total de la deuda pública y privada de corto y largo plazo en dólares, de acuerdo a la metodología del Banco de la Republica de Colombia en el cual no incluye pasivos de corto plazo por inversiones de portafolio en Colombia. (Títulos de deuda pública y acciones adquiridas por inversionistas directos en el mercado local), El flujo neto de la deuda externa no necesariamente corresponde a la variación del saldo, ya que este último incluye el efecto por tasa de cambio, al igual que las variables anteriormente descritas esta fue transformada a logaritmo quedando expresada en elasticidad ${ }^{5}$.

\footnotetext{
${ }^{1}$ Las ponderaciones totales corresponden a la participación móvil de orden 12 de cada país en el comercio exterior colombiano (importaciones y exportaciones) con los 22 principales socios.

${ }^{2}$ Con excepción de Panamá para el que se utiliza el IPC como deflactor.

${ }^{3} \mathrm{Ln}\left(\mathrm{ITCR} \_\mathrm{IPP}\right)=\ln \left(\mathrm{ITCR}=\frac{P * S}{P S *}\right)$

Donde:

$\mathrm{P} *$ : Índice de precios externos

$\mathrm{P}$ : Índice de precios internos

$\mathrm{S}$ : Índice de tasa de cambio interna

S* : Índice de tasa de cambio externa

${ }^{4} \mathrm{Ln}\left(\mathrm{I} \_\mathrm{TDI}\right)=\ln \left(\mathrm{ITI}=\left(\frac{P x}{P m}\right) * 100\right)$

Donde:

Px : precios de las exportaciones locales

Pm : precios de las importaciones

${ }^{5} \mathrm{Ln}\left(\mathrm{I}_{-} \mathrm{TDPYP}\right)=\ln (\mathrm{TDPYP}=(\mathrm{DPc}+\mathrm{DPl}+\mathrm{DPPc}+\mathrm{DPPl}))$ Donde:

DPc : Deuda pública de corto plazo

DPl : Deuda pública de largo plazo

DPPc : Deuda privada de corto plazo

DPPl : Deuda privada de largo plazo
} 


\section{Representación gráfica de las variables}

Las variables representadas en la Gráfica 1. Elasticidad del índice de tasa de cambio real evaluada a través del índice de precios del productor equivalente a los bienes transables de la economía y la Elasticidad del índice de términos de intercambio, tienen un comportamiento inverso entre ellas ampliamente justificado por la teoría económica donde al tenerse incrementos en la tasa de cambio real es decir apreciación de una moneda extranjera sobre la moneda nacional conlleva a una disminución de la relación de intercambio de los bienes en la economía al no poderse adquirir la misma cantidad de bienes en un escenario contrario de depreciación de la moneda extranjera donde la moneda nacional adquiere poder adquisitivo y por ende la adquisición de bienes aumenta es decir los términos de intercambio son favorables para la economía local.

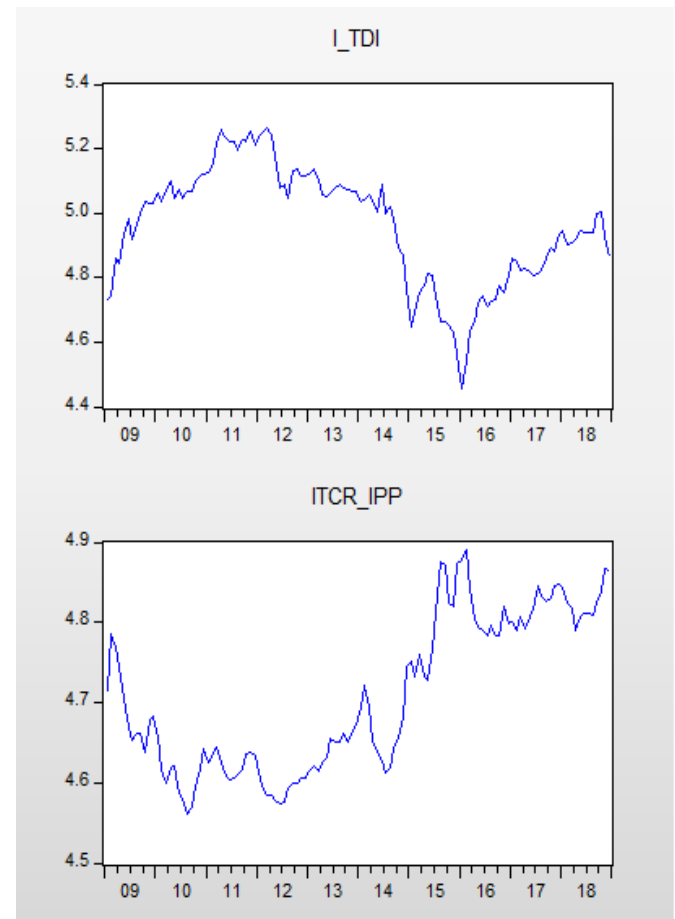

Fuente: Salida del programa estadístico en base a los datos del Banco de la República, DANE

En la Gráfica 2, están representadas las variables Elasticidad del índice de deuda total colombiana en USD y la Elasticidad del índice de términos de intercambio, las cuales mostraran una relación directa dada la Función Impulso-Respuesta, sin embargo existe periodos en el tiempo donde se evidencia una relación inversa como por ejemplo en el mes de diciembre del 2014; la elasticidad del índice de deuda 
total colombiana en USD presenta una tendencia creciente a partir de ese año es decir que de acuerdo a lo que compone esta variable (Banrep) el financiamiento obtenido bajo las modalidades de préstamos bancarios, créditos comerciales o de proveedor, títulos de deuda (bonos) y arrendamiento financiero, se incrementaron, caso contrario ocurrió con la elasticidad del índice de términos de intercambio con los precios de las exportaciones mayores a las importaciones esta disminuyo, convocando a una restructuración en la composición de este índice justificado posiblemente por la recomposición de la deuda en posibles préstamos para capital productivo.

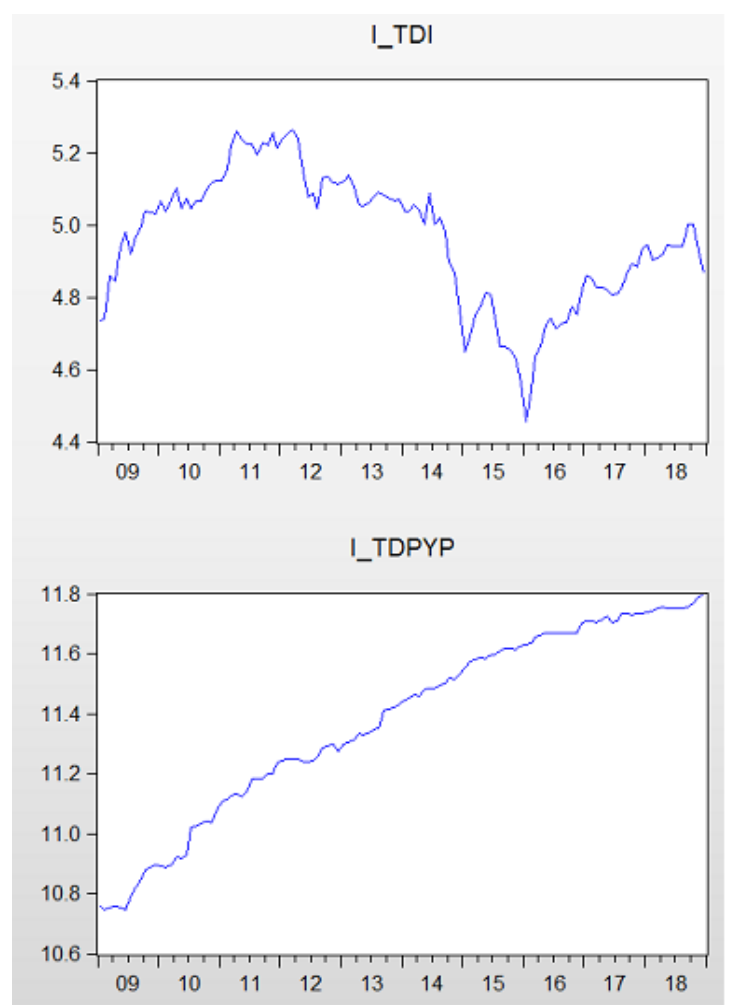

Fuente: Salida del programa estadístico en base a los datos del Banco de la Republica, DANE

\section{Pruebas de raíz unitaria}

En la Tabla 1. se presentan las pruebas de raíz unitaria usando la prueba de Dickey-Fuller Aumentada (DFA), que al comparar con un nivel de significancia de 0.05 se acepta la hipótesis de raíz unitaria por lo cual se procede a aplicar el operador diferencia una vez para cada una de las series. 
Tabla 1. Prueba de Raíz Unitaria

\begin{tabular}{|clc|}
\hline Nombre & Adj. t-Stat & Prob.* \\
I_TDI & -1.551487 & 0.5042 \\
I_TDPYP & -2.459154 & 0.1281 \\
ITCR_IPP & -1.255458 & 0.6484 \\
*MacKinnon & (1996) one-sided p-values. \\
\hline
\end{tabular}

Fuente: Salida del programa estadístico.

En la Tabla 2. se presentan las pruebas de raíz unitaria con una diferencia usando la prueba de Dickey-Fuller Aumentada (DFA), que al comparar con un nivel de significancia de 0.05 no se acepta la hipótesis de raíz unitaria por lo cual las series son integradas de orden 1 (I(1)).

Tabla 2. Prueba de Raíz Unitaria. Primera diferencia

\begin{tabular}{|ccc|}
\hline Nombre & Adj. t-Stat & Prob. $^{*}$ \\
I_TDI & -9.314736 & 0.0000 \\
I_TDPYP & -10.48781 & 0.0000 \\
ITCR_IPP & -8.905125 & 0.0000 \\
*MacKinnon & $(1996)$ one-sided p-values. \\
\hline
\end{tabular}

Fuente: Salida del programa estadístico.

\section{Prueba de cointegración de Johansen}

En la Tabla 3. se presenta las pruebas de cointegración de la traza y máximo valor propio en las cuales bajo el nivel de significancia especificado anteriormente se acepta la hipótesis de cointegración de las variables en estudio. 
Tabla 3. Prueba de Cointegración de Johansen

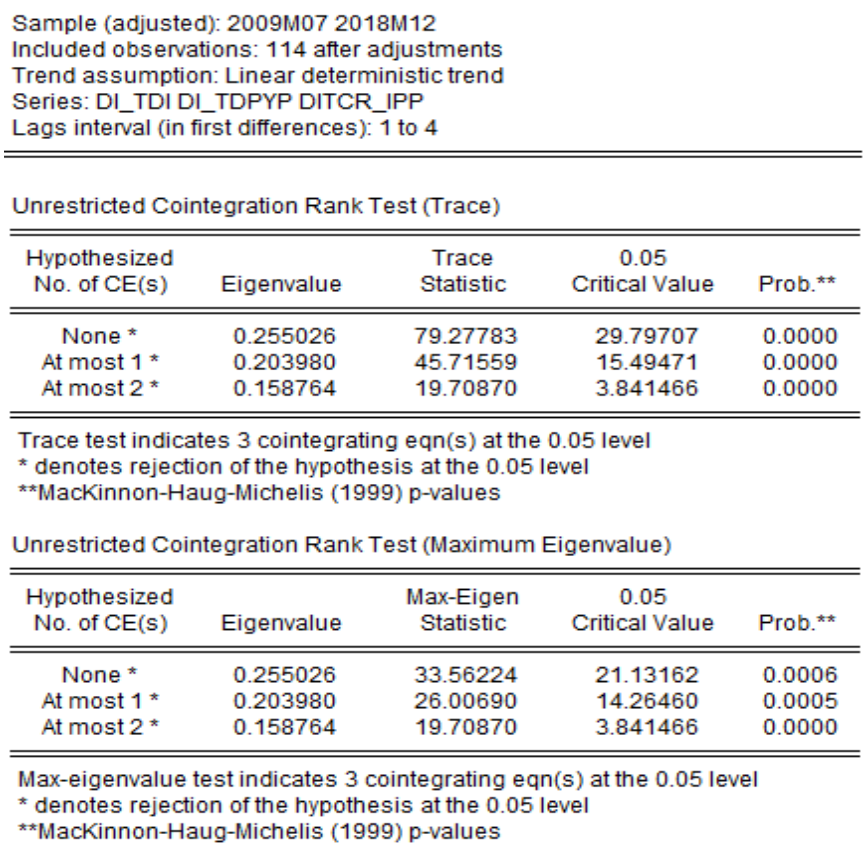

Fuente: Salida del programa estadístico.

\section{Elección del Modelo}

El criterio de información Bayesiana (BIC) propuesto por Schwarz en (1978), ha sido uno de los métodos más populares usado para la selección de modelos. Se basa en la función de probabilidad logarítmica (LLF) y está estrechamente relacionado con el criterio de información de Akaike, ver Tsay (2005).

De acuerdo a lo anteriormente descrito, se encuentra que el mejor modelo es un $\operatorname{VEC}(1)$.

Tabla 4. Selección del modelo usando el criterio BIC

\begin{tabular}{|c|c|}
\hline VEC & BIC \\
1 & $-12,80744$ \\
2 & $-12,73742$ \\
3 & $-12,51682$ \\
4 & $-12,30384$ \\
5 & $-12,18485$ \\
6 & $-11,91306$ \\
\hline
\end{tabular}

Fuente: Elaboración propia.

Comunicaciones en Estaística, diciembre 2019, Vol. 12, No. 2 
En la gráfic 5, se puede apreciar el vector de corrección y el modelo VEC(1), donde el vector de corrección estará dado por $\beta^{\prime}=(1, \quad-1.085072,2.013570,0.006671)$. Este vector presenta equilibrio en el largo plazo por lo tanto se puede representar de la siguiente manera, DITDI $t_{t}-1.085072 D I T D Y P Y_{t}+2.013570 D T C R I P P_{t}=0$ , por ende DITDI ${ }_{t}=1.085072 D I T D Y P Y_{t}-2.013570 D T C R I P P_{t}$, lo que se evaluaría como un incremento en la variable DITDYPY, genera un incremento en la variable DITDI, y un incremento en DTCRIPP ocasiona una disminución en la variable DITDI.

Gráfica 5. Ajuste del modelo VEC (1) para las variables DITDI, DITDYPY, DTCRIPP

\begin{tabular}{|c|c|c|c|c|c|}
\hline \multirow{2}{*}{ 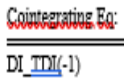 } & \multirow{2}{*}{$\begin{array}{c}\text { CointEq! } \\
1.000000\end{array}$} & \multirow{2}{*}{ Enor Correction: } & \multirow{2}{*}{ D(DI_TDI) } & \multicolumn{2}{|c|}{ D(DI_TDPYD(DITCR_IP } \\
\hline & & & & P) & \\
\hline $\mathrm{DI} \_\mathrm{IDPYP}(-1)$ & $\begin{array}{c}-1.085072 \\
(0.40161) \\
{[-2.70181]}\end{array}$ & CointEq! & $\begin{array}{r}-0.528143 \\
(0.11406) \\
{[-4.63053]}\end{array}$ & $\begin{array}{l}0.074903 \\
(0.04481) \\
{[1.67151]}\end{array}$ & $\begin{array}{c}-0.266942 \\
(0.05492) \\
{[-4.86023]}\end{array}$ \\
\hline DITCR $\mathbb{P D}(-1)$ & $\begin{array}{c}2.013570 \\
(0.24761) \\
{[8.13216]}\end{array}$ & D(DI $\underline{\operatorname{IDI}}(-1))$ & $\begin{array}{l}-0.196509 \\
(0.09540) \\
{[-2.05982]}\end{array}$ & $\begin{array}{r}-0.027540 \\
(0.03748) \\
{[-0.73475]}\end{array}$ & $\begin{array}{l}0.122828 \\
(0.04594) \\
{[2.67365]}\end{array}$ \\
\hline \multirow[t]{4}{*}{ c } & 0.006671 & $D(D I I D P Y P(-1))$ & $\begin{array}{c}-0.021958 \\
(0.22252) \\
{[-0.09868]}\end{array}$ & $\begin{array}{c}-0.396605 \\
(0.08742) \\
{[-4.53756]}\end{array}$ & $\begin{array}{c}-0.332997 \\
(0.10715) \\
{[-3.10766]}\end{array}$ \\
\hline & & $\mathrm{D}\left(\mathrm{DITCR} \_\mathrm{PD}(-1)\right)$ & $\begin{array}{l}0.248734 \\
(0.19621) \\
{[1.26771]}\end{array}$ & $\begin{array}{c}-0.027065 \\
(0.07709) \\
{[-0.35110]}\end{array}$ & $\begin{array}{l}0.006006 \\
(0.09448) \\
{[0.06357]}\end{array}$ \\
\hline & & c & $\begin{array}{r}-0.001586 \\
(0.00430) \\
{[-0.36866]}\end{array}$ & $\begin{array}{c}6.27 \mathrm{~B}-05 \\
(0.00169) \\
{[0.03710]}\end{array}$ & $\begin{array}{l}0.000316 \\
(0.00207) \\
{[0.15246]}\end{array}$ \\
\hline & & \multicolumn{2}{|c|}{ 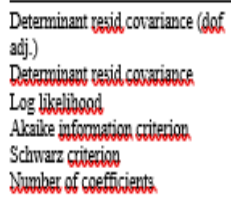 } & $\begin{array}{l}3.01 \mathrm{E} \cdot 10 \\
2.64 \mathrm{E} \cdot 10 \\
792.0948 \\
-13.23239 \\
-12.80744 \\
18\end{array}$ & \\
\hline
\end{tabular}

Fuente: Salida del programa estadístico.

\section{Función Impulso-Respuesta}

La Función Impulso-Respuesta para las variables diferencia del índice de términos de intercambio y diferencia del índice de deuda total de la economía colombiana se presenta en la Gráfica 3. y evidencia una relación positiva ya que la relación entre las exportaciones e importaciones del país genera una reacción en la deuda total que vienen siendo los pasivos contractuales que asumen los residentes como lo son préstamos bancarios, créditos comerciales o de proveedor, títulos de deuda (bonos) y arrendamiento financiero, de los cuales pueden ser para la producción o compra de los bienes importados o exportados. 


\section{Gráfica 3. Función Impulso-Respuesta DITDI - DITDYPY \\ ResponseofDI_TDItoDI_TDPYPInnovation}

usingCholesky(d.f.adjusted)Factors

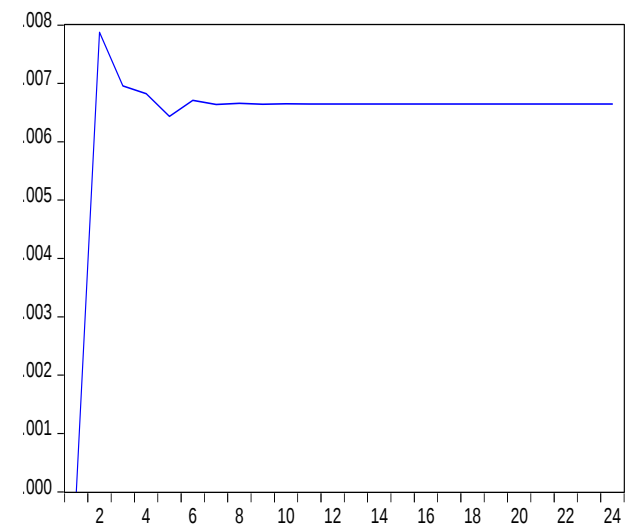

Fuente: Salida del programa estadístico.

Caso contrario ocurre con la Funcion Impulso-Respuesta de las variables diferencia del indice de terminos de intercambio y diferencia de la tasa de cambio real con deflactor indice de precios de productor se presenta en la Gráfica 4. en la cual se evidencia un efecto negativo, ya que una variacion entre las relaciones de los precios de exportación e importación positiva genera una perdida en terminos de valor relativo de la canasta de bienes nacional respecto a los socios comerciales.

Gráfica 4. Función Impulso-Respuesta DITDI - DTCRIPP

ResponseofDI_TDItoDITCR_IPPInnovation

usingCholesky(d.f.adjusted)Factors

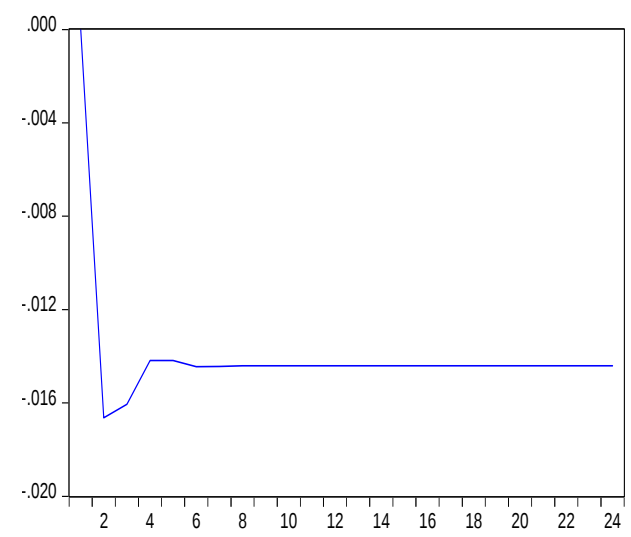

Fuente: Salida del programa estadístico.

Comunicaciones en Estaística, diciembre 2019, Vol. 12, No. 2 


\section{Validación del modelo}

\section{Normalidad multivariada}

La Tabla 6. muestra que los residuales multivariados no siguen una distribución normal multivariada, pero como lo indica ((Lutkepohl 2005)) donde la comprobación de estas cantidades tiene sentido si se desea realizar pronósticos a través de intervalos de confianza, al igual que ((Price. et al. 2003)) donde indican que es más importante que el modelo cumpla con la prueba de errores no autocorrelacionados que con la normalidad múltiple.

Tabla 6. Prueba de normalidad residual VEC VEC Residual Normality Tests Orthogonalization: Cholesky (Lutkepohl)

Null Hypothesis: Residuals are multivariate normal

\begin{tabular}{|c|c|c|c|}
\hline & Jarque- & & \\
\hline Component & Bera & $\mathrm{Df}$ & Prob. \\
\hline 1 & 3.571665 & 2 & 0.1677 \\
\hline 2 & 109.4646 & 2 & 0.0000 \\
\hline 3 & 1.139202 & 2 & 0.5658 \\
\hline Joint & 114.1755 & 6 & 0.0000 \\
\hline
\end{tabular}

*Approximate p-values do not account for coefficient Estimation

Fuente: Salida del programa estadístico.

\section{Prueba de Autocorrelación}

En el Gráfico 5. y Tabla 7. se presentan los auto-correlogramas y la prueba de Auto correlación en los cuales se evidencia que existe independencia con un nivel de significancia de 0.05 . 
Tabla 6. Prueba de normalidad residual VEC AutocorrelationswithApproximate2Std.Err.Bounds

$\operatorname{Cor}(\mathrm{DI}+T D I, D I+T D I(-i))$

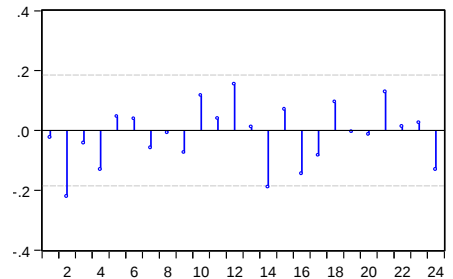

Cor(DI_TDPYP,DI_TDI(-i))

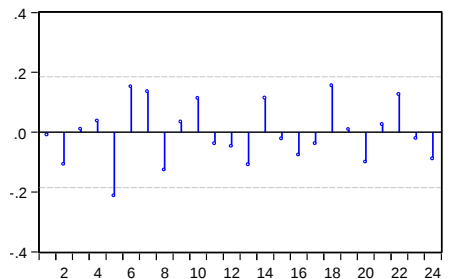

$\operatorname{Cor}(\mathrm{DITCR}$ IPP,DI_TDI(-i))

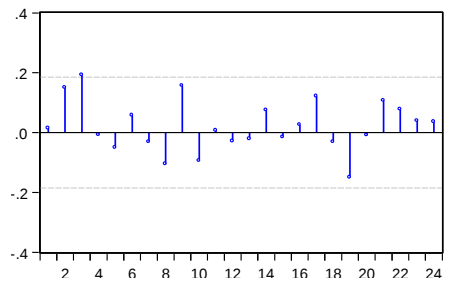

Cor(DI_TDI,DI_TDPYP(-i))

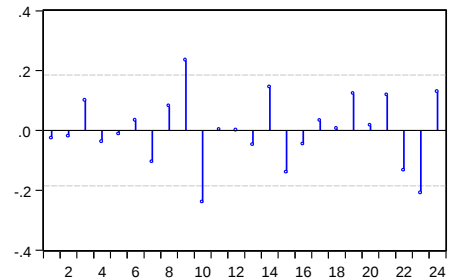

$\operatorname{Cor}\left(D I \_T D P Y P, D I / T D P Y P(-i)\right)$

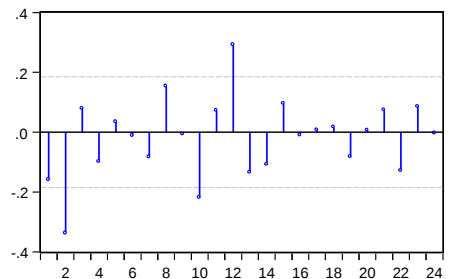

Cor(DITCR_IPP,DI_TDPYP(-i))

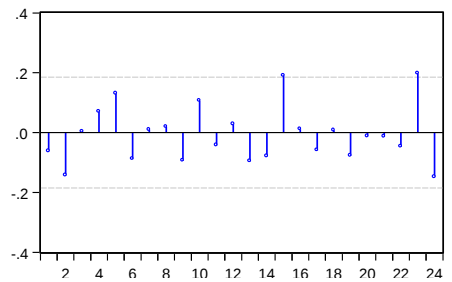

Cor(DI_TDI,DITCR_IPP(-i))

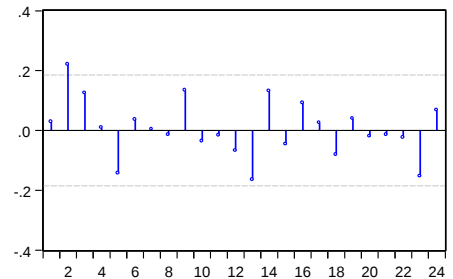

Cor(DI_TDPYP,DITCR_IPP(-i))

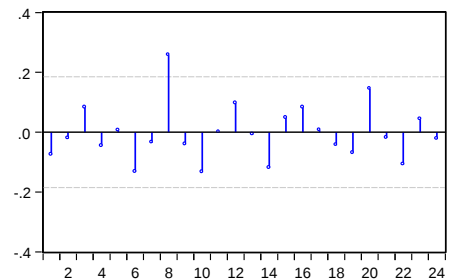

$\operatorname{Cor}\left(D I T C R \_I P P, D I T C R \_I P P(-i)\right)$

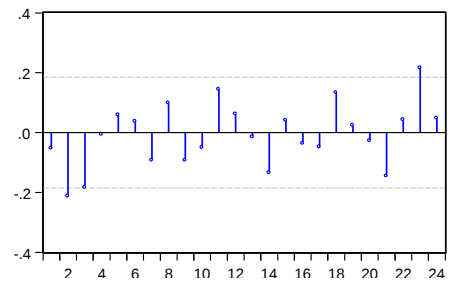

Fuente: Salida del programa estadístico. 
Tabla 7. Prueba de Auto correlación residual

\begin{tabular}{|c|c|c|c|c|c|c|}
\hline \multicolumn{7}{|c|}{ Null hypothesis: No serial correlation at lag h } \\
\hline Lag & LRE* stat & df & Prob. & Rao F-stat & df & Prob. \\
\hline 1 & 26.47986 & 9 & 0.0017 & 3.053765 & $(9,260.6)$ & 0.0017 \\
\hline 2 & 36.84238 & 9 & 0.0000 & 4.334704 & $(9,260.6)$ & 0.0000 \\
\hline 3 & 9.924980 & 9 & 0.3566 & 1.108867 & $(9,260.6)$ & 0.3567 \\
\hline 4 & 5.224456 & 9 & 0.8143 & 0.578505 & $(9,260.6)$ & 0.8144 \\
\hline 5 & 11.18201 & 9 & 0.2634 & 1.252306 & $(9,260.6)$ & 0.2635 \\
\hline 6 & 6.837254 & 9 & 0.6541 & 0.759415 & $(9,260.6)$ & 0.6541 \\
\hline 7 & 7.940368 & 9 & 0.5402 & 0.883790 & $(9,260.6)$ & 0.5402 \\
\hline 8 & 12.23113 & 9 & 0.2006 & 1.372543 & $(9,260.6)$ & 0.2007 \\
\hline 9 & 10.80455 & 9 & 0.2893 & 1.209163 & $(9,260.6)$ & 0.2894 \\
\hline 10 & 19.63883 & 9 & 0.0203 & 2.235260 & $(9,260.6)$ & 0.0203 \\
\hline 11 & 5.842066 & 9 & 0.7556 & 0.647653 & $(9,260.6)$ & 0.7557 \\
\hline 12 & 13.90179 & 9 & 0.1259 & 1.565004 & $(9,260.6)$ & 0.1259 \\
\hline 13 & 10.25782 & 9 & 0.3300 & 1.146781 & $(9,260.6)$ & 0.3301 \\
\hline 14 & 11.63090 & 9 & 0.2349 & 1.303693 & $(9,260.6)$ & 0.2350 \\
\hline 15 & 6.323429 & 9 & 0.7072 & 0.701658 & $(9,260.6)$ & 0.7072 \\
\hline 16 & 4.414965 & 9 & 0.8820 & 0.488119 & $(9,260.6)$ & 0.8821 \\
\hline 17 & 2.842341 & 9 & 0.9702 & 0.313313 & $(9,260.6)$ & 0.9702 \\
\hline 18 & 6.274384 & 9 & 0.7122 & 0.696151 & $(9,260.6)$ & 0.7122 \\
\hline 19 & 6.784527 & 9 & 0.6595 & 0.753483 & $(9,260.6)$ & 0.6596 \\
\hline 20 & 3.618173 & 9 & 0.9347 & 0.399421 & $(9,260.6)$ & 0.9347 \\
\hline 21 & 10.12440 & 9 & 0.3405 & 1.131578 & $(9,260.6)$ & 0.3406 \\
\hline 22 & 9.602286 & 9 & 0.3836 & 1.072155 & $(9,260.6)$ & 0.3837 \\
\hline 23 & 16.61490 & 9 & 0.0551 & 1.880161 & $(9,260.6)$ & 0.0551 \\
\hline 24 & 9.032076 & 9 & 0.4343 & 1.007393 & $(9,260.6)$ & 0.4344 \\
\hline
\end{tabular}

Fuente: Salida del programa estadístico.

\section{Conclusiones}

Al realizarse el análisis del comportamiento de las variables utilizadas en el modelo para Colombia en los periodos comprendidos entre enero-2009 y diciembre- 2018, el mejor modelo para evaluar la intensidad de la interacción de las variables es un $\operatorname{VEC}(1)$.

Existe una relación inversa entre las variables Elasticidad del índice de tasa de cambio real evaluada a través del índice de precios del productor equivalente a los bienes transables de la economía y la Elasticidad del índice de términos de intercambio, al tenerse incrementos en la tasa de cambio real es decir apreciación de una moneda extranjera sobre la moneda nacional conlleva a una disminución de la relación de intercambio de los bienes en la economía al no poderse adquirir la misma cantidad de bienes. En caso contrario de depreciación de la moneda extranjera donde la moneda nacional adquiere poder adquisitivo y por ende la adquisición de bienes aumenta es decir los términos de intercambio son favorables para la economía local, la relación es justificada en un análisis preliminar y en la Función Impulso-Respuesta planteado. 
Hay una relación positiva entre la Elasticidad del índice de términos de intercambio respecto a la Elasticidad del índice de la deuda total colombiana, la relación entre las exportaciones e importaciones del país genera una reacción en la deuda total que vienen siendo los pasivos contractuales que asumen los residentes como lo son préstamos bancarios, créditos comerciales o de proveedor, títulos de deuda (bonos) y arrendamiento financiero, de los cuales pueden ser para la producción o compra de los bienes importados o exportados.

Recibido: Septiembre de 2019 Aceptado: Febrero de 2020

\section{Referencias}

Andrade, L., Martínez, V., da Conceição Rebuge, E. \& González, J. (2007), 'Una aplicación de la metodología VAR al ámbito del marketing periodístico: el caso de la promoción de ventas.', Revista Electrónica de Comunicaciones $Y$ Trabajos de ASEPUMA 8, 151-162.

Arteaga, C., Granados, J. \& Ojeda, J. (2012), 'El comportamiento del tipo de cambio real en colombia: ¿explicado por sus fundamentales?', Borradores de Economia 742, 1-35.

Bigio, S. \& Salas, J. (2006), Efectos no lineales de choques de política monetaria y de tipo de cambio real en economías parcialmente dolarizadas: un análisis empírico para el perú., Technical report, Banco central de Peru.

Calderón, C. (2006), Un análisis del comportamiento del tipo de cambio real en chile., Technical report, Banco Central de Chile.

Clavijo, P., Regules, J. \& Bogliaccini, J. (n.d.), Terminos de intercambio y salario real, in 'XX jornadas anuales de Economia'.

Echavarría, J., López, E. \& Misas, M. (2009), 'Intervenciones cambiarias y política monetaria en colombia. un análisis de var estructural', Borradores de Economia 580, 1-37.

Fernández, H. (2013), La influencia del tipo de cambio real en la inversión e innovación de las pequeñas y medianas empresas (pymes) en santander 2003-2012., Technical report, Universidad Santo Tomas.

Garavito, A., López, D. \& Montes, E. (2011), 'Aproximación a los índices de valor unitario y quantum del comercio exterior colombiano.', Borradores de Economia 680, 1-29.

Licandro, G. \& Masoller, A. (2000), 'La composición óptima por monedas de la deuda pública uruguaya.', Revista de Economía 7, 135-180. 
Lutkepohl, H. (2005), New introduction to multiple time series analysis, Springer, Berlin [u.a.].

Misas, M., . P. C. (2000), 'Crecimiento y ciclos económicos en colombia en el siglo xx: el aporte de un var estructural.', Borradores de Economia 155, 1-53.

Modelización Econométrica Multiecuacional Modelo De Vectores Cointegrados (Vec) Y Teoría De Cointegración (n.d.).

*http://ucv-rodolfomedina.blogspot.com.co/2015/01/econometria-ii.html

Otero, J. (1997), 'Los determinantes de la tasa de cambio real en Colombia.', Coyuntura Económica 7, 1-12.

Pindyck, R. \& Rubinfeld, D. (2001), Econometría : modelos y pronósticos, McGraw-Hill, México D.F.

Price, S. \& Blake, A. (2003), The dynamics of consumers? expenditure: the uk consumption ecm redux, Technical report, Bank of England.

Rodríguez, H. (2011), 'Estudio del fenómeno de inflación importada vía precios del petróleo y su aplicación al caso colombiano mediante el uso de modelos var para el periodo 2000-2009.', Estudios Gerenciales 27, 79-97.

Sims, C. (1980), 'Macroeconomics and reality', Econometrica 48(1), 1-48.

Tsay, R. (2005), Analysis of Financial Time Series, John Wiley \& SONS. 